\title{
Coordinated observational campaigns for non-radially pulsating objects
}

\author{
K. R. Pollard, ${ }^{1}$ D. J. Wright, ${ }^{1}$ P. L. Cottrell, ${ }^{1}$ R. M. Woollands, ${ }^{1}$ D. J. Ramm, ${ }^{1}$ T. Böhm ${ }^{2}$ \\ ${ }^{1}$ Department of Physics and Astronomy, University of Canterbury, Christchurch 8020, New Zealand \\ ${ }^{2}$ Laboratoire Astrophysique de Toulouse, Observatoire Midi-Pyrenees, Toulouse, France
}

\begin{abstract}
In recent years we have initiated and contributed to a number of campaigns to study nonradially pulsating objects. Our observing facility is the Mt John University Observatory $1.0 \mathrm{~m}$ telescope equipped with a high-efficiency and extremely stable echelle spectrograph, ideal for spectroscopic mode identification. Our current interests include $\delta$ Scuti star campaigns and a programme to study the non-radial pulsations in $\gamma$ Dor stars. We are investigating several different methods of line profile analysis and spectroscopic mode identification of these targets. An overview of the programme, with specific examples, is presented.
\end{abstract}

\section{Spectroscopy at the Mt John University Observatory}

The instrumentation for asteroseismology at the University of Canterbury's Mt John University Observatory (MJUO) is the $1.0 \mathrm{~m}$ telescope with the fibre-fed High Efficiency and Resolution Canterbury University Large Echelle Spectrograph, HERCULES $(R \approx 40000$ or 80000 ; Hearnshaw et al. 2002;). The major elements of HERCULES are fixed to an optical bench located inside a cylindrical vacuum tank $(4.3 \times 1.2 \mathrm{~m})$ in which the pressure is maintained at 1 to 5 torr. The tank is situated in a thermally isolated and insulated room. RMS stability of $15 \mathrm{~m} \mathrm{~s}^{-1}$ over time spans of 4 to 5 years is being achieved. This is ideal for high-resolution, time-series asteroseismological studies of reasonably bright stars $(V<9)$. The longitude of MJUO, coupled with our ability to acquire long sequences of observing time using this facility, allow us to coordinate and contribute to both single-site and multi-site asteroseismology campaigns.

\section{Target stars, analysis techniques and results}

We have completed the observational aspect of one multi-site campaign on QW Pup and HD 139095 (Wright et al. 2006) and are undertaking single-site observations from MJUO of a larger list of targets (Table 1 ). We have measured projected rotational velocities, identified binary or multiple systems and are investigating line-profile variations (LPV). A number of our targets have turned out to be in multiple stellar systems and orbital periods are still being determined.

Line profiles are tested for variation by visual inspection of stacked plots and by plotting the residuals after subtraction of the average line profile. To increase our sensitivity to small-scale line profile variations, a high $\mathrm{S} / \mathrm{N}$ representative line profile is obtained through cross correlating selected lines in each spectrum. Techniques used to analyse the line profile variations include the moment method (Briquet \& Aerts 2003) and the phase change across the profile method (Telting \& Schrijvers 1997). Our intention is to carry out spectroscopic mode identification by comparing the observed line profile variations with those predicted from models of the various non-radially pulsation modes. 
Table 1: Targets observed using the MJUO $1.0 \mathrm{~m}$ and HERCULES.

\begin{tabular}{|llll|}
\hline Star & Comments & \# obs & $V \sin i\left(\mathrm{~km} \mathrm{~s}^{-1}\right)$ \\
\hline HD 10167 & FOV, $V=6.676$, SB2 & 2 & $6 \pm 2,6 \pm 2$ \\
HD 14940 & FOIV, $V=6.673, \gamma$ Dor & 2 & $44 \pm 2$ \\
HD 17310 & F0, $V=7.79$ & 2 & $7 \pm 3$ \\
HD 27377 & FOV, $V=7.4$ & 1 & $8 \pm 2$ \\
HD 40745 & F2IV $, V=6.207, \gamma$ Dor & 2 & $40 \pm 2$ \\
HD 41448 & A9V, $V=7.6$ & 2 & $106 \pm 5$ \\
HD 75747 & A7V, $V=6.07$, RS Cha, SB2 & 351 & $69 \pm 2,72 \pm 2$ \\
HD 166114 & F2V, $V=5.858$, Triple system? & 19 & $8 \pm 2,7 \pm 2$ \\
HD 172416 & F5V, $V=6.632$, SB1 & 10 & $54 \pm 3$ \\
HD 187028 & FOV, $V=7.5, \gamma$ Dor & 1 & $95 \pm 7$ \\
HD 189631 & FOV, $V=7.54$, LPV & 7 & $51 \pm 5$ \\
HD 214291 & F7V, $V=6.581$, SB2 & 78 & $69 \pm 3,69 \pm 3$ \\
HD 216910 & F2IV, $V=6.699, \gamma$ Dor, LPV & 11 & $100 \pm 6$ \\
\hline
\end{tabular}

\section{References}

Briquet M., Aerts C., 2003, in Sterken C., ed., ASP Conf. Ser. Vol. 292, Interplay of Periodic, Cyclic and Stochastic Variability in Selected Areas of the H-R Diagram. Astron. Soc. Pac., San Francisco, p. 365

Hearnshaw J. B., Barnes S. I., Kershaw G. M., et al., 2002, Experimental Astronomy, 13, 59

Telting J. H., Schrijvers C., 1997, A\&A, 317, 723

Wright D. J., Pollard K. R., Cottrell P. L., 2006, Mem. Soc. Astron. Ital., 77, 490 